Muséologies

Les cahiers d'études supérieures

muséologies

\title{
De l'intégrité de l'oeuvre d'art afin d'assurer sa juste pérennité : un entretien mené par Justine Lebeau avec Richard Gagnier, restaurateur en art contemporain
}

\section{Justine Lebeau}

Volume 5, numéro 1, automne 2010

URI : https://id.erudit.org/iderudit/1033526ar

DOI : https://doi.org/10.7202/1033526ar

Aller au sommaire du numéro

Éditeur(s)

Association Québécoise de Promotion des Recherches Étudiantes en Muséologie (AQPREM)

ISSN

1718-5181 (imprimé) 1929-7815 (numérique)

Découvrir la revue

Citer ce document

Lebeau, J. (2010). De l'intégrité de l'oeuvre d'art afin d'assurer sa juste pérennité : un entretien mené par Justine Lebeau avec Richard Gagnier, restaurateur en art contemporain. Muséologies, 5(1), 180-209.

https://doi.org/10.7202/1033526ar d'utilisation que vous pouvez consulter en ligne. 
DEUX

De l'intégrité de l'œuvre d'art afin d'assurer sa juste pérennité : un entretien mené par Justine Lebeau avec Richard Gagnier, restaurateur en art contemporain

ENTREVUE RÉALISÉE LE 18 MARS 2010

Richard Gagnier occupe actuellement le poste de chef du service de restauration au Musée des beaux-arts de Montréal. Détenteur d'un baccalauréat en chimie et d'une mineure en histoire de l'art de l'Université de Montréal, il a également complété la scolarité en restauration-conservation à l'Université Queen's à Kingston, Ontario. Il a occupé les postes d'assistantrestaurateur et restaurateur de l'art contemporain au Musée des beaux-arts du Canada de 1984 à 2007. A titre de chercheur, il participe à de multiples projets de recherche (dont l'Alliance DOCAM de la Fondation Daniel Langlois), en plus de coordonner le chapitre nord-américain de l'International Network for the Conservation of Contemporary Art.

Justine Lebeau termine actuellement une maîtrise en études des arts à l'université du Québec à Montréal portant sur la réactualisation des collections fermées. Depuis 2008, elle assume le poste d'auxiliaire de recherche pour une étude présidée par Francine Couture portant sur la réexposition, la réactualisation et la pérennité des cuvres contemporaines. En 2009, elle a participé également à la recherche pour le modèle documentaire développé par DOCAM à la Fondation Daniel Langlois. JUSTINELEBEAU@GMAIL.COM 


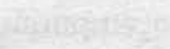

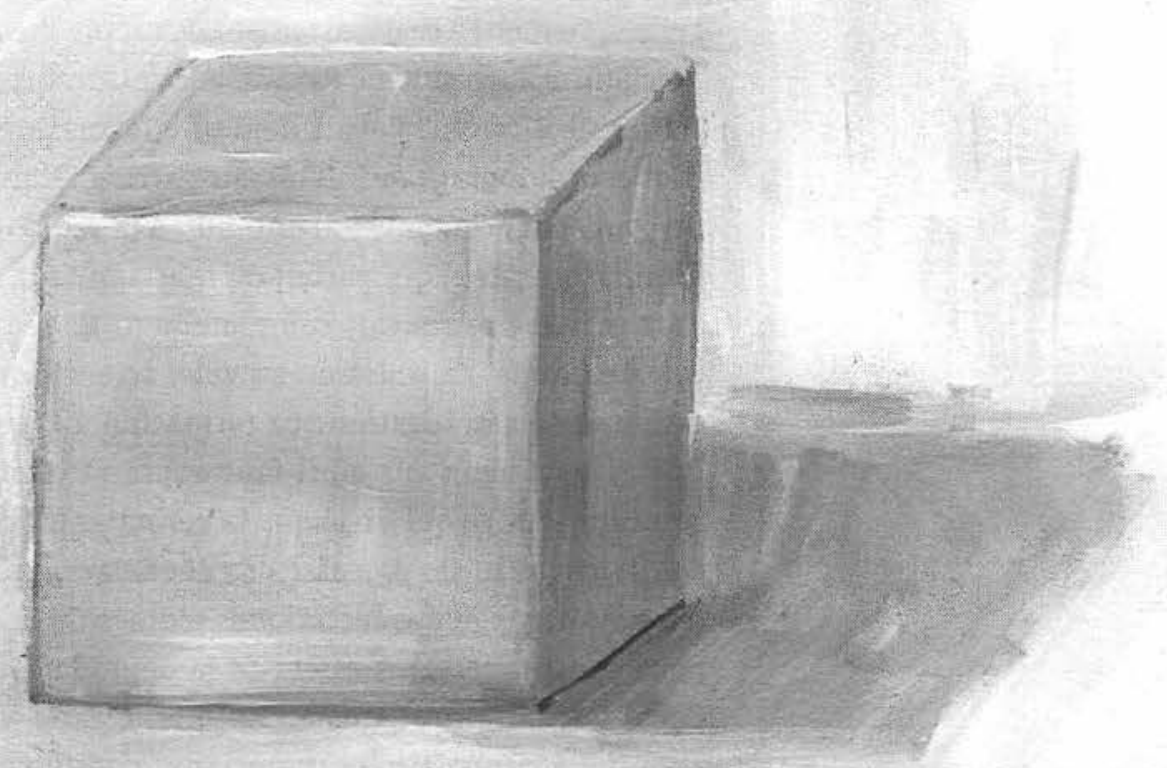




\section{Justine Lebeau}

Pourriez-vous nous parler de votre formation universitaire et en quoi elle a pu vous aider pour la pratique de la restauration en art contemporain?

\section{Richard Gagnier}

Comme la plupart des canadiens qui veulent devenir restaurateur d'œuvre d'art, la formation principale est la maîtrise à l'Université Queen's de Kingston. Au premier cycle, j'ai étudié en sciences, complétant un baccalauréat en chimie. J'ai ensuite fait des études en histoire de l'art, en me spécialisant sur les discours et les pratiques de l'art contemporain à l'Université de Montréal. C'était la grande époque de l'enseignement à ce département des approches structurales avec, entre autres, René Payant, Alain Laframboise et Nicole Dubreuil-Blondin. J'avais déjà une formation générale et l'art contemporain m'intéressait particulièrement. Et puis un jour, par accident, j'ai entendu à la radio une entrevue d'un restaurateur qui parlait du mariage de la science et de l'art et je me retrouvais complètement là-dedans. J'ai fait une demande d'admission au volet scientifique de ce programme de restauration. La formation à Queen's est une maîtrise de deux ans où il faut apprendre le métier de la restauration, ce qui implique la connaissance de la nature des matériaux, des processus de dégradation sous différentes conditions et des techniques mêmes de la restauration pour contrer certains effets de la dégradation du temps. Cette formation est très technique, c'est-à-dire que l'on apprend à connaître les matériaux, comment une œuvre d'art est constituée, comment elle se fabrique, et cela dans le contexte de l'histoire des technologies. A travers la formation, on nous parle de déontologie, des manières d'aborder l'œuvre d'art, du respect de son authenticité et du lien intrinsèque de cette notion avec les matériaux d'origine. De ce contexte découle le principe de la réversibilité des interventions pour le métier. On se rend compte dans le métier de la restauration qu'il y a eu des interventions dans le passé qui ont été trop excessives, qui ont causé des dommages permanents et la perte littérale ou la modification irréversible des matériaux originaux. Le principe de la réversibilité est donc extrêmement important. 
Au regard des auteurs auxquels on se réfère, on nous parlait des concepts explicités par Cesare Brandi, sans qu'il ne soit souvent nommé'. Il y avait quand même un cours de déontologie/méthodologie où on lisait certains auteurs afin d'apprendre et d'être à l'affût de ce qui a été écrit sur la restauration. Y était discutées, entre autres, les grandes controverses qui ont marqué en peinture l'application des vernis et leur enlèvement, du point de vue historique de leur technologie. Par ces discussions, on arrivait à situer les écoles de pensée récentes, par exemple la pensée nord-américaine versus l'approche européenne, leur position par rapport au vernis synthétique et naturel. Finalement, on apprenait les principes de base, c'est-à-dire à pratiquer dans la mesure du possible des interventions minimales qui soient réversibles, permettant des interventions futures moins drastiques si les conditions de l'œuvre se détériorent à nouveau.

Cela dit, le métier de la restauration s'apprend encore beaucoup dans le rapport maître à élève, par le travail avec des restaurateurs aguerris. La formation continue de la restauration est bien marquée par les conférences annuelles qui s'organisent entre différentes organisations professionnelles comme l'IIC (International Institute for conservation), l'ICOM (International Council of Museums) et l'ICC (Institut canadien de conservation).

À Queen's, les restaurateurs sont formés en peinture, en papier et en objet, cette dernière spécialité comprenant les objets ethnographiques, archéologiques, la sculpture et le mobilier. A l'époque où j'ai reçu ma formation, tous les étudiants - quel que soit le choix de leur spécialité - devaient suivre l'intégralité des cours sur les matériaux et leurs technologies, ce qui n'est plus le cas aujourd'hui. En ce qui me concerne et pour la suite avec l'art contemporain, cette approche s'est avérée extrêmement profitable.

\section{Justine Lebeau}

Est-ce que la culture classique du restaurateur est encore utile en regard des pratiques caractérisant l'art contemporain, par exemple avec les cuvres de type allographique ou les pratiques de l'installation?
1 BRANDI, Cesare. Théorie de la restauration. Paris: Édition du patrimoine, Monum, 2001, $207 \mathrm{p}$. 


\section{Richard Cagnier}

Même en art contemporain, la question de l'authenticité est encore très liée, dans une perspective de pérennité, à celle des matériaux originaux, à la possibilité ou non de leur maintien et à l'exploration de nouvelles solutions selon les œuvres et leurs problèmes particuliers de détérioration. L'enjeu ultime de la restauration, qui est un peu une fiction, est de maintenir la condition des matériaux au moment où on les trouve, c'est-à-dire à ce moment historique d'une certaine dégradation, d'un usage et des anciennes interventions qui ont été faites.

Quand je suis arrivé à la restauration au début des années 1980, il y avait une mouvance d'intérêt pour la pratique de la restauration en art contemporain. De plus en plus de restaurateurs commençaient à se spécialiser dans ce domaine. Il y avait des collections d'art contemporain qui se formaient ; c'était une problématique qui apparaissait quelque peu différente de la restauration traditionnelle, générant un questionnement sur les notions de l'authenticité et de l'originalité des matériaux. Beaucoup d'auteurs commençaient à réfléchir non seulement aux problèmes structuraux des tableaux de grands formats, mais aussi à l'apparition de méthodes de fabrication mécanique et industrielle dans la production de l'œuvre, à l'insertion de produits de consommation de façon plus ou moins modiflée et aux particularités des cuvres polymorphes et hybrides comme l'installation. Le métier de la restauration est lié aux pratiques de l'art; il s'ajuste à la nature des objets, d'autant plus dans le cas de l'art contemporain produit à partir de la deuxième moitié du $\mathrm{XX}^{\mathrm{e}}$ siècle.

C'est intéressant de voir la spécialisation nécessaire du domaine, de se rendre compte que des concepts peuvent déontologiquement être examinés sous un angle autre que la restauration traditionnelle. Cela permet de modifier les méthodes tout en incluant dans l'analyse du problème cette possibilité de la sauvegarde des matériaux originaux de l'œuvre. Traditionnellement en restauration, lorsqu'on parle d'authenticité de l'objet, on signifie que celui-ci, malgré son vieillissement et les interventions effectuées, 
doit maintenir sa matérialité, preuve de son existence vérifiée. Ce qui revient aussi à dire que l'intégrité est maintenue, celle-ci étant encore fortement liée à la matérialité originale de l'objet. Ainsi, en peinture, tous les constituants d'une œuvre sur un panneau de bois ou sur la toile font que l'objet est intègre même si le vieillissement de certains pigments ou du vernis modifie l'équilibre original des tonalités, résultat d'un jaunissement ou d'un obscurcissement par oxydation. Par exemple, Poussin utilisait certains verts composés de sels de cuivre qui se sont oxydés et qui ont bleui. Aujourd'hui, on perçoit des feuillages aux tons bleutés dans ses peintures et on peut comprendre que ces pigments étaient en fait verts. Ce processus agit dorénavant comme une espèce de garantie de l'authenticité de l'œuvre, preuve connue de vieillissement observé chez Poussin. Ce sont ces marques du temps qui, chez Brandi, constituent ce qu'il appelle "l'unité potentielle de l'objet ", c'est-àdire la saisie qui est faite de l'objet à un moment donné.

Je vous parle beaucoup de restauration traditionnelle, mais le parcours de la déontologie en art contemporain est lié à celui de l'art ancien. Même en art ancien, cette volonté de maintenir l'authenticité des matériaux a subi des distorsions dans la pratique de la restauration, ne serait-ce que par la pratique acceptée du dévernissage des tableaux, admettant donc la perte d'un matériau original. Aujourd'hui, comme suite aux écrits de Brandi et à ceux d'auteurs américains, allemands et britanniques notamment, cette notion de l'authenticité en rapport à la stricte référence aux matériaux d'origine s'est quelque peu modifiée, mais l'idée de "l'unité potentielle de l'œuvre » est quelque chose qui a été très prégnant et qui a marqué le domaine de la restauration depuis les années 1960.

\section{Justine Lebeau}

Il semble donc que la diversité et le développement des pratiques de l'art contemporain viennent ébranler et questionner la nature même de ce qu'est l'œuvre d'art, ainsi que les conditions qui permettent sa manifestation. Pourriez-vous nous parler plus en détails de certaines pratiques qui viennent ébranler le paradigme de 


\section{conservation muséologique et conséquemment celui de la restauration?}

\section{Richard Gagnier}

Effectivement, cette observation est symptomatique de bien des pratiques de l'art contemporain et va influencer l'approche de gestion des collections. Avec la deuxième moitié du $\mathrm{XX}^{\mathrm{e}}$ siècle, l'artiste questionne son cadre de production, le statut reconnu de celui qui fabrique, transforme et produit l'objet d'art à partir de matériaux bruts. Les artistes re-questionnent non seulement les limites de la pratique de l'art, mais aussi les limites de l'art sous forme d'un objet non équivoque, permanent. Ainsi avec l'art conceptuel, l'œuvre peut apparaître comme un processus à faire et à fabriquer, ce qui implique que la collection, cette entité matérielle des œuvres au sein de l'institution, doit s'ouvrir à la possibilité d'admettre une certaine dématérialisation de l'objet. Un exemple classique serait les Wall Drawings de Sol LeWitt. LeWitt développe conceptuellement sa pratique en reprenant, au cours de sa carrière, beaucoup de matériaux et de procédures qui appartiennent au domaine du dessin, très souvent complétés in situ. Ses projets sont déployés à même le mur, souvent à grande échelle. Pour ce faire, il s'entoure d'un groupe de jeunes artistes qui les réalisent, sous sa direction, pour les institutions acquéreuses. Or, les Wall Drawings existent seulement au moment de leur itération, c'est-à-dire le temps de leur période d'exposition. Ils sont éventuellement effacés du mur choisi. Comme objet collectionné, ils sont une méthode d'application, précise et documentée avec leur liste de matériaux. Ils sont à refaire. Il y a donc des pratiques de l'art contemporain, différentes de l'art ancien, où l'adéquation de la déontologie de la restauration prenant. comme principe la matérialité d'origine à préserver ne peut avoir sa raison d'être. Ce modèle atteint sa limite.

Le cas des installations pose autrement ce problème. En effet, elles sont produites à partir de multiples éléments, physiques, technologiques, lumineux qui prennent en charge un espace et modifient l'expérience de l'œuvre. Ces éléments peuvent être réarrangés selon la volonté de 
l'artiste et présentés dans des configurations différentes d'une fois à l'autre. Un passage très important se produit lorsque ce type d'œuvre est acquis par l'institution et entre dans la collection. Par le travail de documentation, le restaurateur cherche à établir la stabilité de l'objet, mais d'une façon non réductrice. La responsabilité passe alors de l'artiste aux professionnels du musée. Le contexte et les possibilités de faire des variations, d'intervenir régulièrement sur l'objet devront être clairement définis, bien entendu en discussion avec l'artiste, parce que l'on souhaite maintenir le caractère authentique de l'objet, mais aussi assurer la possibilité de présenter l'œuvre sans l'aide de l'artiste, en dehors de son droit de l'altérer ou d'en modifier le devenir. Grâce au travail de documentation et surtout aux entretiens avec l'artiste, il faut déterminer les variations possibles de présentation et établir un protocole, le "script " de l'œuvre dira Poinsot ${ }^{2}$. Ce "script " définit les limites d'existence de l'œuvre par rapport à ses constituants et à ses possibilités de mise en espace.

L'œuvre Tap (1969) (ill. 1, 2, 3) de Michael Snow de la collection đu Musée des beaux-arts du Canada est intéressante au regard de cette notion de script puisqu'elle rend compte des incidents d'usage de l'œuvre et montre la possibilité de variance. Initialement, Tap est constituée par le son généré et enregistré par l'artiste qui tapote sur un micro. La matérialité du son est uniquement signifiee par la présence d'un haut-parleur déposé au sol à une certaine distance du mur, le lecteur à bande magnétique $1 / 8$ de pouce étant dissimulé à la vue du spectateur. L'œuvre est également constituée d'une photographie noir et blanc, de dimensions moyennes, où l'on voit les mains et les bras de l'artiste tapotant sur le micro au-dessus de l'enregistreuse à ruban qui capte la prise du son. Il y a de plus un objet encadré - un texte tapé à la machine sur papier - où Snow explique le processus de production de l'œuvre et cornment elle devrait être présentée en ses divers éléments. L'artiste demande que ces éléments soient éparpillés en plusieurs endroits du lieu d'exposition, probablement pour marquer l'idée que le son se déploie librement et que dans sa matérialité l'œuvre n'est pas unitaire. Cette œuvre a été acquise par le musée
2 POINSOT. Jean-Marc. Quand locuvre a lieu: Part exposé et ses récits autorisés. Genève: Editions du Musée d'art moderne et contemporain. Villeurbanne: Editions de l'Institut d'art contemporain, 1999, 328 p. 


\section{Illustration 1}

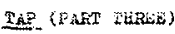

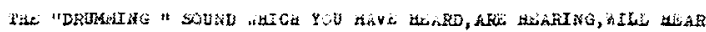

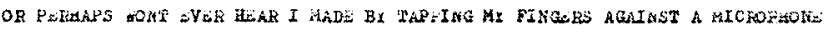

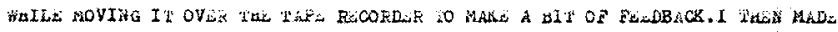

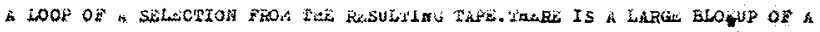

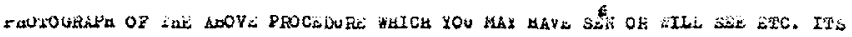

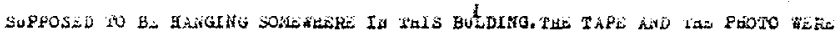

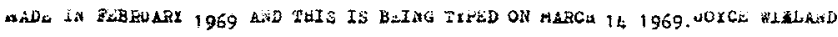

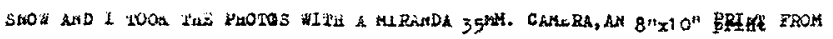

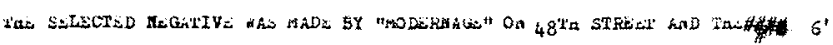

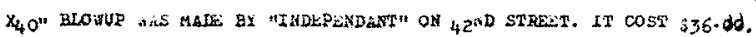

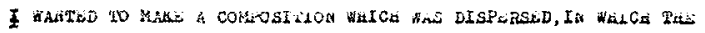

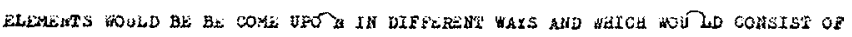

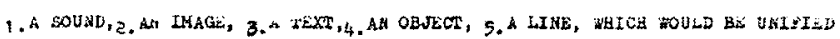

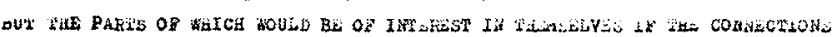

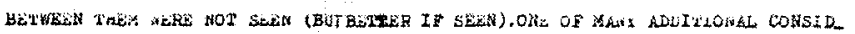

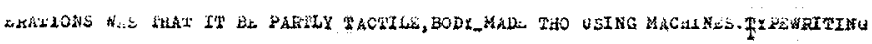

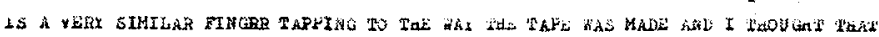

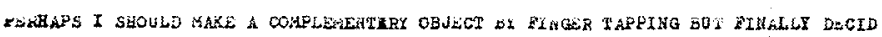

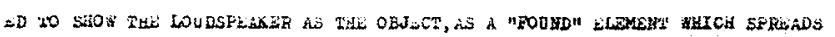

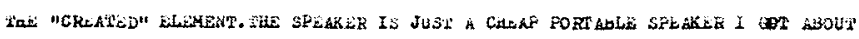

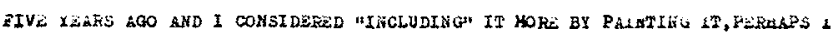

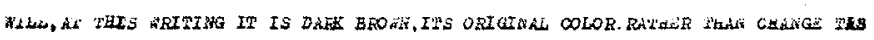

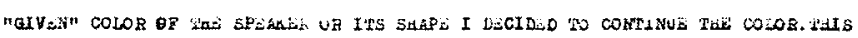

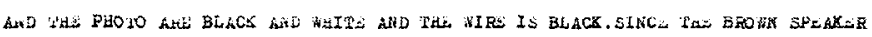

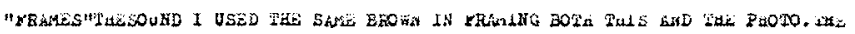

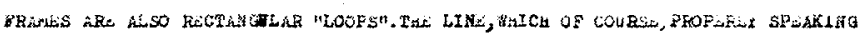

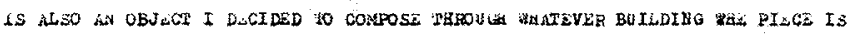

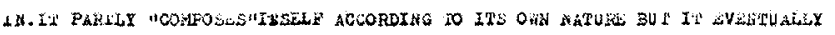

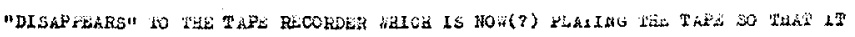

(6) W

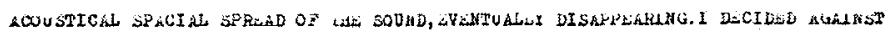

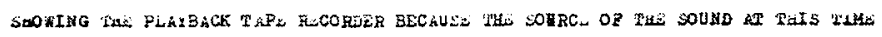

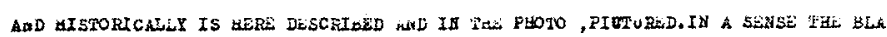

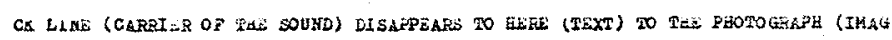

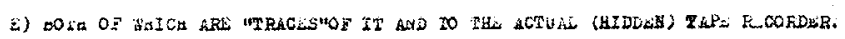
THË PILCE $Z$ I L

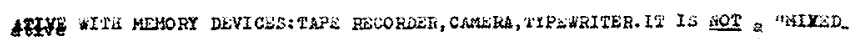

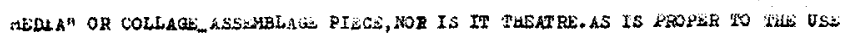

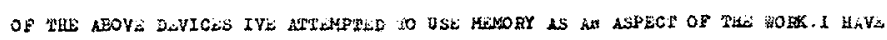

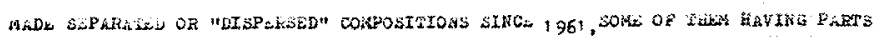

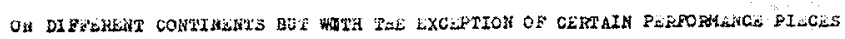

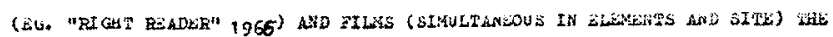

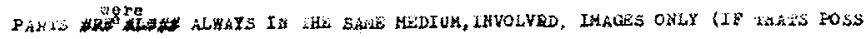
1BLE) OR WOKED IN AN IMAEE TO OBNLCT SCALE.

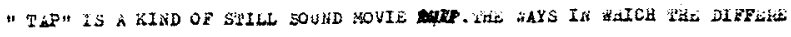

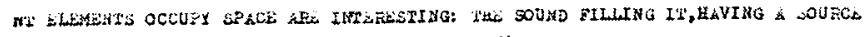

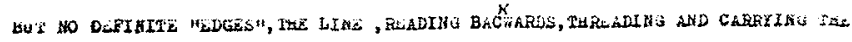

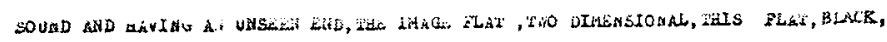

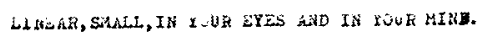

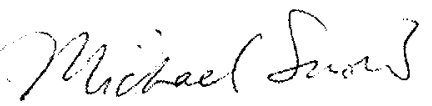




\section{Illustration 2}

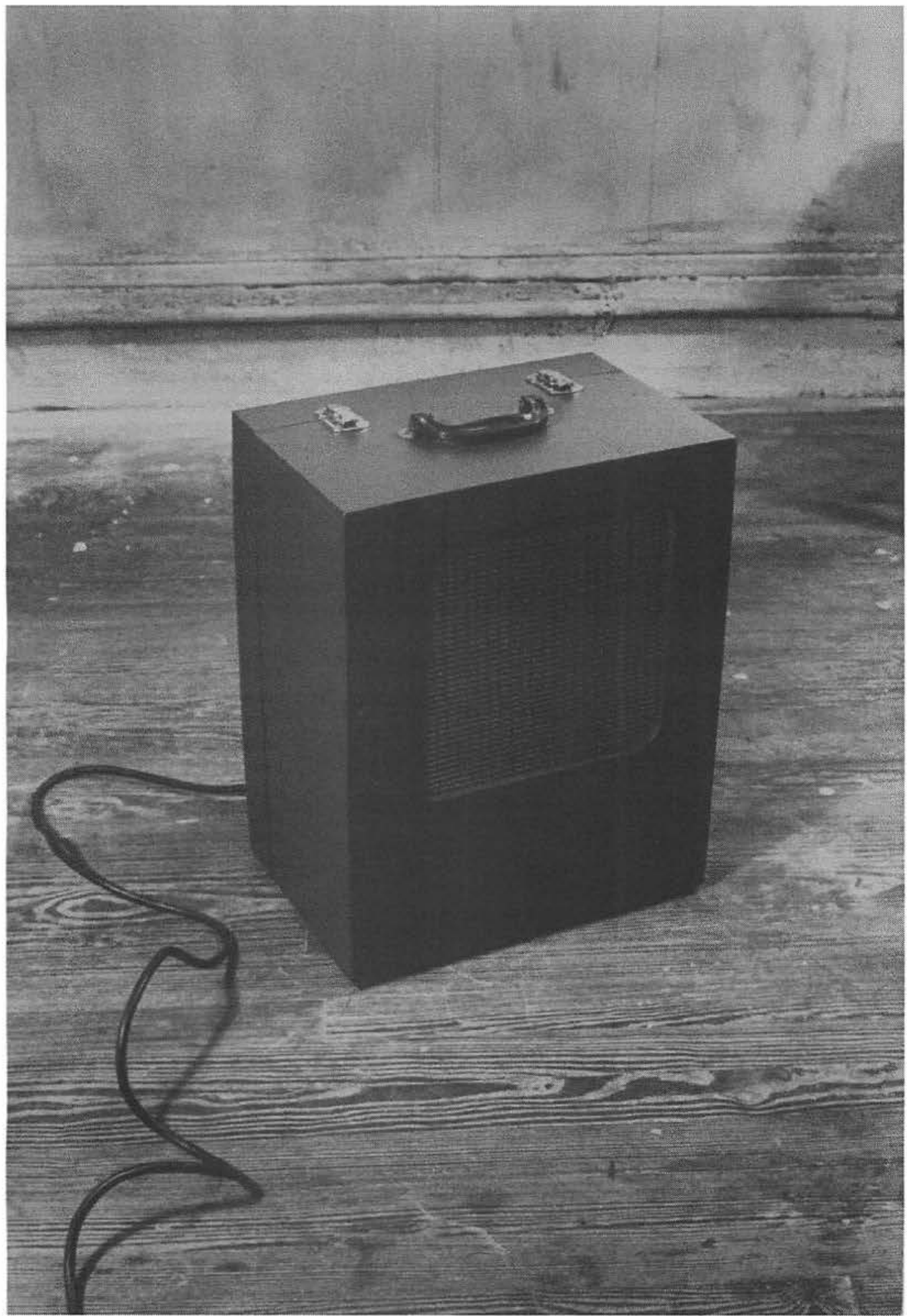




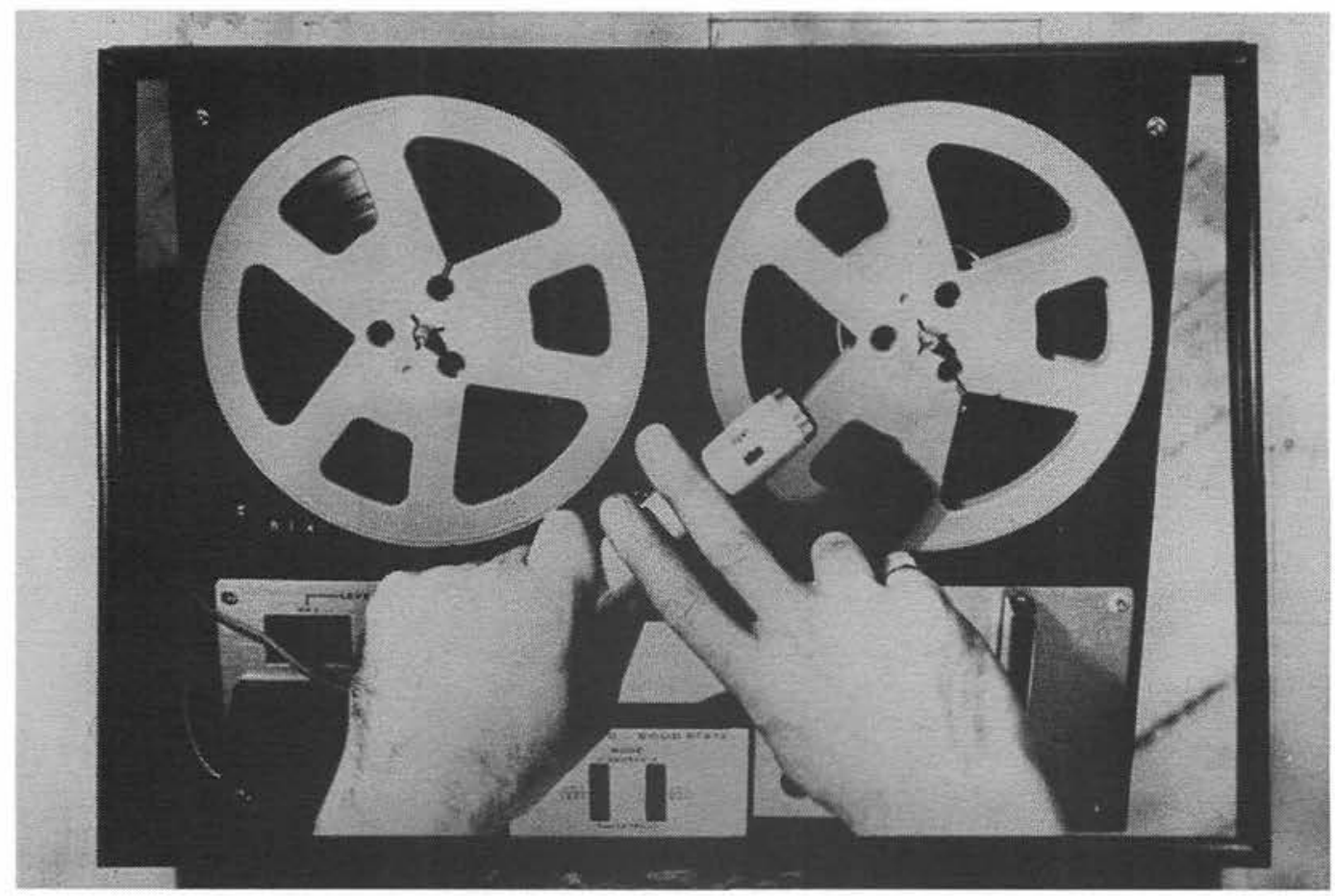

\section{Illustrations 1, 2, 3}

Michael Snow, Tap, 1969-1972, photographies en noir et blanc encadrées, texte dactylographié sur papier encadré, magnétophone, haut-parleur, fil et bande audio, photographie encadrée : 106,8 x $156,3 \mathrm{~cm}$, texte encadré : $65,1 \times 40$ $\mathrm{cm}$; haut-parleur: $48,3 \times 38,1 \times$ $25,4 \mathrm{~cm}$. Musée des beaux-arts du Canada, Ottawa. Photo: $O$ MBAC 
au début des années 1970. Quelques années plus tard, on a voulu présenter l'œuvre à nouveau et on s'est aperçu que le haut-parleur avait disparu, sans doute confondu pour un objet usuel. Le conservateur a écrit à l'artiste pour l'informer de cette perte et lui demander d'en envoyer un semblable, de même modèle, afin de maintenir l'intégrité de l'œuvre. Cette possibilité de remplacer le haut-parleur de fabrication industrielle devenait parfaitement acceptable. Snow, dans sa façon de penser l'ceuvre, a répondu que ce serait les techniciens du musée qui le construiraient. Il a envoyé un devis technique où il spécifiait les mesures du haut-parleur à construire, ses matériaux, de même qu'il informait le conservateur qu'il devrait ajouter un nouvel élément encadré, selon les mêmes modalités de présentation que les autres cadres. Il a demandé de documenter par une photographie un moment de la construction du haut-parleur en présence des techniciens. Dans le nouveau cadre, il a demandé de faire apparaître la lettre officielle du musée expliquant la perte de l'objet, le dessin technique qu'il a soumis, la photographie de construction, de même que sa réponse explicative. Poursuivant l'entreprise conceptuelle de mise en représentation du processus de l'œuvre, Snow ajoutait ainsi un nouveau trait à l'œuvre: Tap sera désormais constituée de cet ajout documentaire. Nous appuyant sur Gérard Genette, nous pourrions alors dire qu'il y a eu un "remaniement" de l'œuvre et que, à partir de là, Tap a atteint sa présentation "correcte" telle que le musée doit la faire perdurer ${ }^{3}$. Ce qui fascine, c'est que son parcours muséologique récent est introduit dans la définition de l'œuvre. La matérialité première de cette installation est quelque chose d'immatériel, c'est-à-dire que le son constitue l'essence même de l'œuvre; mais ce qui est mis à voir c'est aussi le processus de fabrication de l'œuvre, ses mécanismes de manifestation.

Du point de vue du restaurateur, il faut assurer la stabilité des différents supports qui constituent Tap, dont le papier et la photographie qui ont des procédures de préservation bien établies. Mais qu'en est-il de l'élément sonore? La bande magnétique originale est-elle matériellement stable? Pour nombre de technologies, produire des copies est
3 GENETTE, Gérard. L'ceuvre de l'art: immanence et transcendance. Paris: Seuil, coll. * Poétique», 1994, 299 p. 
possible. Cette approche de la copie permet d'identifier le support original comme source maîtresse de contenu à préserver sous des conditions d'entreposage optimales, source à partir de laquelle des copies d'exposition et d'usage seront produites. Cependant, avec le temps, certaines technologies de support ne sont plus disponibles, y compris celles du médium sonore. D'où la nécessité d'envisager un passage vers une technologie plus récente - la migration - qui devra être soupesée et documentée. Il faudra se poser des questions à chacune des migrations : est-ce qu'il s'ensuit une perte d'information ou une modification par rapport à l'original? Les copies sont-elle vraiment identiques à l'original? Comment évaluer ces pertes? Cette approche est-elle acceptable parce qu'il n'y a pas d'autres moyens de faire perdurer cet objet dans le temps? Est-ce que les équipements pour faire jouer ces supports existent toujours et vont eux aussi perdurer? Tap illustre bien, par son contenu technologique, l'étendue des phénomènes de matérialité autre et nouveau de l'œuvre d'art contemporain. Aujourd'hui, le support sonore de présentation de Tapest un $C D$, le son étant toujours diffusé par le haut-parleur reconstruit.

Cette manifestation temporaire et limitée de la matérialité de l'objet trouve un écho avec la question de l'éphémère en art. Il existe dans l'histoire des pratiques contemporaines des artistes qui réfléchissent sur le transitoire et le temporaire en travaillant avec des matériaux organiques et des aliments qui vont subir un processus de dégradation. Ces artistes mettent en jeu la détérioration. Certaines institutions reconnaissent l'importance de ces pratiques en acquérant des objets qui contrecarrent la raison d'être de l'objet en muséologie, c'est-à-dire sa pérennité. Cependant, ces œuvres peuvent avoir comme prescription une activation du processus de détérioration à chacune des itérations. Elles deviennent pérennes par la documentation qui est faite de leurs méthodes de fabrication et de leurs matériaux constituants. Il est important de reconnaître ce caractère de reprise. Je pense qu'en fait il y a très peu d'œuvres qui ont été pensées pour marquer le fait inéluctable du transitoire, c'est-à-dire qui se vouent à l'anéantissement. Très souvent, c'est plutôt sa documentation qui fera office de l'œuvre 
pour la collection. Advenant la possibilité que ce type d'objet entre dans la collection, la responsabilité du restaurateur serait, à mon avis, de contrôler le processus de dégradation par des mesures environnementales comme l'abaissement de la température en réserve, permettant ainsi de prolonger l'existence de l'cuvre sans stopper sa dégradation physico-chimique. Je ne crois pas que cela aille à l'encontre du souhait et des principes conceptuels qui dictent l'œuvre.

La déontologie de la restauration en art contemporain nécessite le savoir combiné du conservateur du musée, du restaurateur et de l'artiste vivant. Celui-ci nous fait des commentaires sur les matériaux utilisés et leur signification. Il peut également nous indiquer sa position par rapport au vieillissement et à la pérennité de son œuvre. Il y a des institutions qui décident d'assumer l'importance d'une production éphémère parce qu'elle systématise des enjeux, des concepts, un effet de culture à un certain moment. Lors de l'acquisition d'une œuvre, il devrait y avoir un premier entretien où l'artiste définit les matériaux renouvelables et à préserver, la construction, l'agencement, les conditions de présentation, toutes ces considérations de l'œuvre qui, comme pour l'installation, sont des paramètres qui n'existent pas dans la production traditionnelle. De plus, en documentant un corpus, il est souhaitable de produire un deuxième ou un troisième entretien où l'artiste va alors nous parler de l'ensemble de sa production. Lorsqu'on produit dans le temps des entretiens, on se rend compte que certains concepts vont devenir prépondérants ou seront perçus de façon différente par l'artiste, alors que d'autres devienđront négligeables.

La responsabilité du restaurateur est d'assurer une pérennité à partir de modalités différentielles selon les œuvres, comme j'ai tenté de le montrer à l'aide de ces quelques exemples. On prend par rapport à l'œuvre des décisions qui respectent les intentions de l'artiste, mais qui tendent à saisir ce qui doit être absolument maintenu de sa matérialité originale ou renouvelable afin d'assurer l'authenticité pérenne de l'objet. 


\section{Justine Lebeau}

Cette matérialité immuable à préserver étant ébranlée, quels seraient les concepts qui permettent encore d'assurer la pérennité de l'œuvre d'art avec ces pratiques à partir du moment où elles sont acquises et entrent au musée?

\section{Richard Gagnier}

Jusqu'à maintenant j'ai souligné quelques aspects qui caractérisent certaines pratiques de l'art contemporain et qui ébranlent, comme vous le dites, le paradigme de la matérialité originale de l'objet garantissant son authenticité. On doit admettre que des œuvres puissent présenter un caractère variable et que, selon les matériaux dont se compose l'œuvre, des protocoles différents de préservation s'élaborent peu à peu. Ces protocoles décisionnels sont influencés, entre autres, par la source des matériaux utilisés, ceux-ci pouvant être des objets trouvés de consommation, de production industrielle ou technologique, mais encore transformés de façon singulière par l'artiste. Je pense ici au travail traditionnel du peintre ou du sculpteur, ces dernières interventions ayant un caractère unique à considérer. Pour l'œuvre, on dira souvent que sa manifestation est "correcte" si son intégrité est maintenue malgré les modifications apportées et les effets du temps. Ce concept est souvent lié à celui de l'authenticité et, parfois, on les utilise de façon quasi-interchangeable. Ce terme peut aider à distinguer les singularités et à nous assurer que nous sommes dans un régime authentique pour ces pratiques de l'art contemporain qui exacerbent le statut et l'existence de l'objet.

Le concept d'intégrité vise au maintien d'une vérité de l'œuvre là où le concept d'authenticité strictement lié au maintien des matériaux originaux de l'œuvre ne peut plus la définir. En restauration d'art contemporain, on a parfois tendance à dire que l'approche de Brandi ne tient plus. Je ne suis pas tout à fait d'accord. Même en art contemporain, il y a une filiation possible, un effet de continuité avec sa pensée, mais elle présente des limites que certaines pratiques de l'art contemporain révèlent. Pour moi les deux termes sont imbriqués. De mon point de vue je préfère 


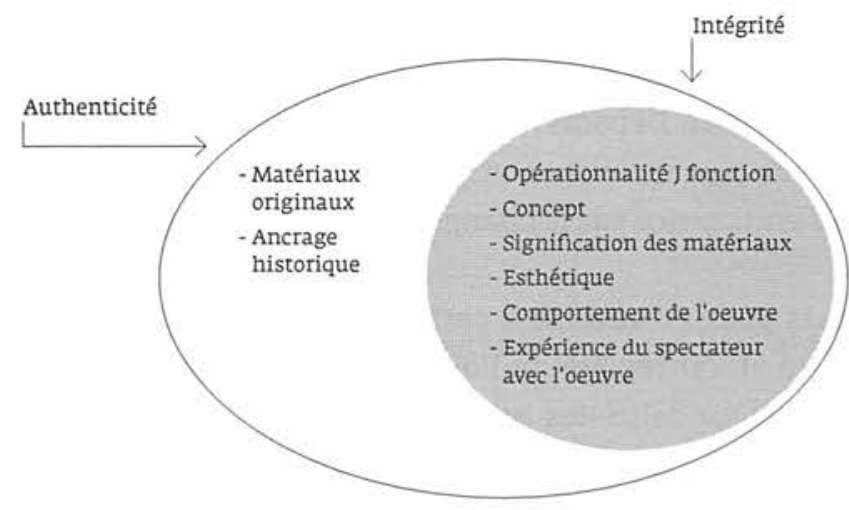

considérer que l'authenticité relève toujours de l'originalité des matériaux, mais qu'elle s'accomplit aussi en d'autres termes. De cette manière l'authenticité rend compte de toutes les caractéristiques de l'œuvre qui maintiennent son intégrité et offrent en plus la possibilité d'assurer la pérennité des matériaux d'origine. L'intégrité, quant à elle, concerne le concept de l'œuvre, le caractère d'opérationnalité ou de fonction (par exemple l'existence d'un mouvement mécanique ou l'inclusion d'équipement technologique), le comportement et les effets désirés induisant un changement au cours de l'itération, la signification que les matériaux apportent au concept et enfin l'esthétique. C'est en identifiant et en mesurant leur importance que l'on parvient à élaborer un protocole de conservation afin d'assurer la juste pérennité de l'œuvre.

Cette réflexion et cette distinction entre le concept d'authenticité et d'intégrité, l'un incluant l'autre, ont émergé du travail de recherche que j'ai mené ces dernières années avec le groupe de recherche du projet DOCAM (Documentation et conservation du patrimoine des arts médiatiques) dirigé par la Fondation Daniel Langlois, projet qui a examiné les problèmes liés à la conservation et à la préservation des œuvres à contenu technologique (ill. 4). Il fallait arriver à bien définir non seulement ce qui de ces œuvres est essentiel à leur portée de sens, mais sous quelles conditions certains composants parviennent toujours à en rendre compte de façon juste dans la durée. Cette recherche est dans la lignée des premiers travaux que cette même
Illustration 4

Diagramme élaboré dans le cadre du projet de recherche DOCAM (documentation et préservation de œuvres à contenu technologique), comité préservation-restauration, Fondation Daniel Langlois pour l'art, la science et la technologie. o Richard Gagnier, 2009 


\section{4}

FONDATION LANCLOIS POUR LART, LASCIENCE ET LATECHNOLOGIE, SOLOMON R. GUGGENHEIM FOUNDATION, Alain DEPOCAS, JON IPPOLITO, Caitlin JONES et al., La permanence par le changement: l'approche des médias variables, New York: Guggenheim Museum Publications, Montréal: Fondation Daniel Langlois pour l'art, la science et la technologie, 2003, 137 p. fondation a entrepris au musée Guggenheim à New York avec, entre autres, Carol Stingari, Caitlin Jones, Jon Ippolito et Alain Depocas, et qui ont mesuré le caractère variable des œuvres tout en affirmant que l'œuvre peut assurer sa permanence par le changement ${ }^{4}$.

Mais pour en revenir à cette distinction entre l'authenticité et l'intégrité de l'objet, l'exemple qui me vient à l'esprit est celui des ceuvres composées de matériaux de consommation trouvés dans le commerce. Le Musée des beaux-arts du Canada possède un tableau du peintre Cary Lee Nova intitulé The Ballad of the Curious and Wise Wizard (1966) (ill. 5). Il s'agit d'une acrylique sur toile de format hexagonal bien représentative de l'esthétique du pop au Canada. Le tableau a la particularité d'intégrer en surface un relief de polystyrène moulé sous vide d'un cornet đe crème glacée - la glace rose sur cône de biscuit, figure familière servant d'enseigne à un grand nombre de bars laitiers, sous lequel une ampoule incandescente de faible intensité clignote de façon continuelle. Cette œuvre est entrée très tôt dans la collection, en 1966, l'année de sa production. A l'époque, le restaurateur qui n'était pas particulièrement formé en art contemporain s'est interrogé sur la durée de cet ajout, ces objets de polystyrène étant très minces et faciles à perforer. Suspectant une instabilité physico-chimique possible du polymère à moyen terme menant à la formation de fissures, il se demandait si l'ampoule sous le moulage ne pourrait pas créer une accumulation de chaleur qui accélérerait la dégradation du plastique, mais qui pourrait aussi causer sa décoloration. Fait intéressant, cet élément étranger fut identifié comme porteur de risque de vieillissement différent, potentiellement plus vulnérable que le film de peinture à l'acrylique, et ce, sous les mêmes conditions de présentation et d'entreposage. Étant donné que le cône est illuminé, toute fissure serait difficile à masquer sans compromettre la translucidité de l'objet et, conséquemment, son intégrité spécifique. Mesurant cette difficulté qui aurait un impact sur l'esthétique du tableau, le restaurateur a alors contacté l'artiste afin de se procurer un certain nombre de ces moulages, en fait quatre, annexés en réserve à la collection permanente. Si l'on revient au modèle déontologique 
de Brandi, le premier cône devrait perdurer avec le tableau quelles que soit les circonstances. Mais le fait que cet élément se retrouvait facilement sur le marché à cette époque a permis de considérer son remplacement futur advenant, par exemple, un acte de vandalisme ou une mauvaise manutention. Le polystyrène est un polymère relativement stable, si bien que cette attitude est tout à fait défendable, les cônes pouvant maintenir leur intégrité matérielle en réserve. A ce jour, c'est toujours le cône original placé par l'artiste qui coiffe le tableau. Dans ce cas, il apparaît plus pertinent de le considérer sous l'angle de l'intégrité, physique, esthétique et même fonctionnelle, pour ce qui sera de remplacer l'ampoule incandescente par un modèle qui, à défaut d'être identique, devra du moins maintenir la même intensité lumineuse. De nos jours, elle sera sans doute remplacée par une ampoule de type DEL (diode électroluminescente), mais, comme elle est dissimulée, l'apparence globale de l'objet n'en sera pas affectée. C'est cette distinction en ce qui concerne l'authenticité qui permet au restaurateur de remplacer des éléments qui sont de l'ordre allographique ou, en ce qui concerne les œuvres technologiques, de remplacer certains équipements défectueux ou obsolètes et de migrer les supports de contenu de l'œuvre.

\section{Justine Lebeau}

Comment le restaurateur d'art contemporain détermine-t-il la façon dont l'œuvre à caractère technologique devra être conservée et restaurée?

\section{Richard Gagnier}

Comme je l'ai expliqué brièvement pour la partie sonore de Tap, ce qui distingue les composants technologiques de tout autre composant de facture industrielle, c'est le fait qu'ils deviennent rapidement désuets, l'industrie cessant leur production au profit d'équipement similaire plus performant ou tout simplement par abandon de cette technologie. On pourrait donner l'exemple des projections vidéographiques basées sur l'usage des disques laser dont la technologie fut remplacée en moins de dix ans, au cours des années 1990, par le DVD. L'obsolescence de ces composants est le problème critique, tant pour les supports de 


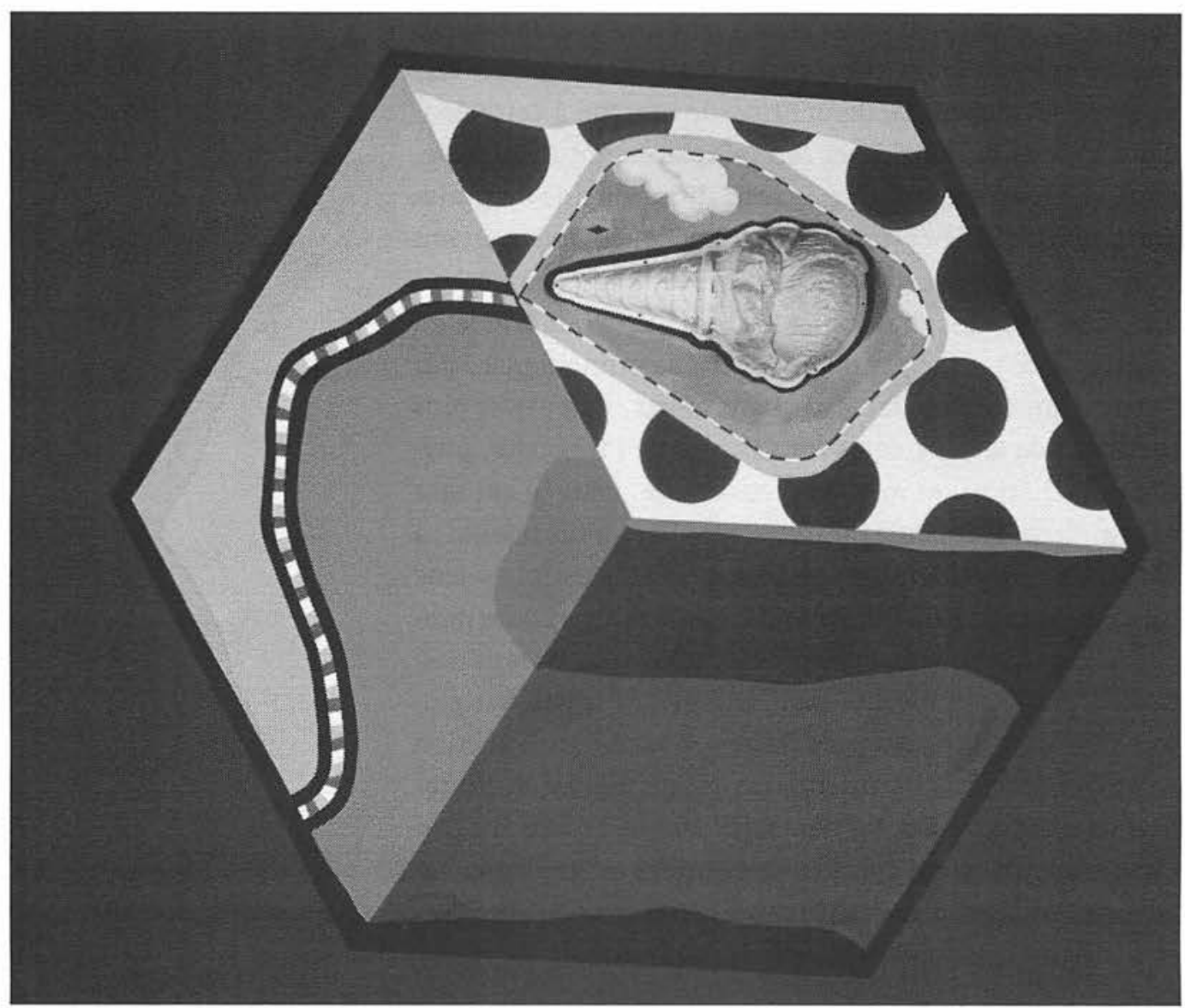

\section{Illustration 5}

Gary Lee Nova, The Ballad of a Wise and Curious Wizard, acrylique sur toile avec enseigne de plastique illuminée, en forme de cornet de crème glacée, 176,2 X 152,2 cm. Musée des beaux-arts du Canada, Ottawa. Photo: o MBAC 


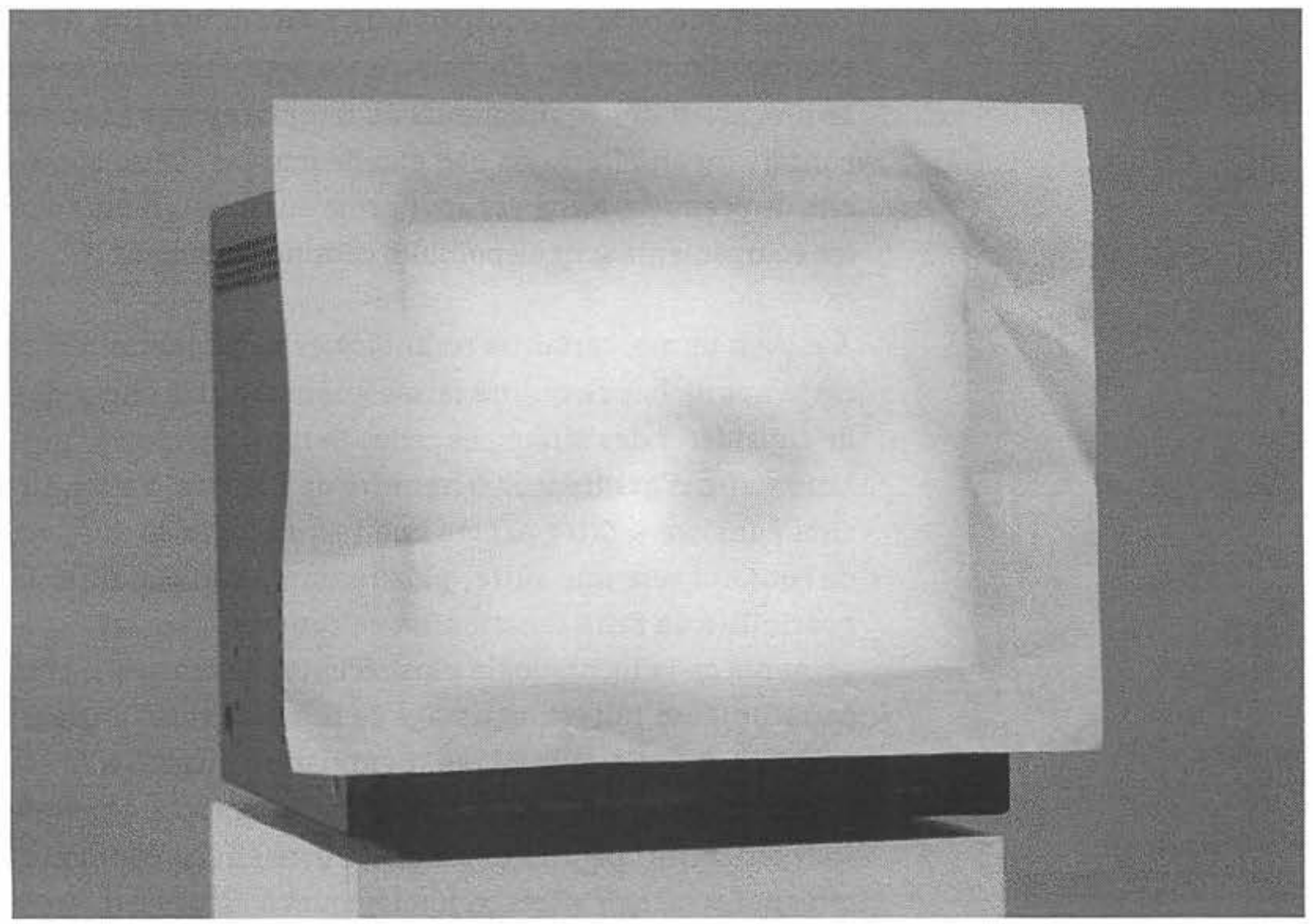

\section{Illustration 6}

Jacques Perron, Défilement, 1997.

bande vidéo transférée sur vidéo-

disque, moniteur, papier, socle,

$1 / 3,154,8 \times 22,3 \times 33 \mathrm{~cm}$ (socle et

moniteur), @ Musée des beaux-arts

de Montréal. Photo: Brian Merrett 
contenu que pour les équipements pouvant les faire fonctionner. En principe, l'obsolescence peut être contrée en se procurant des équipements de remplacement et en les conservant en réserve en bon état de marche. Cette approche de première ligne est applicable aussi longtemps que les équipements sont disponibles commercialement.

A moyen terme, certaines technologies ne permettent plus cette approche, ce qui ne laisse guère d'autre choix que de considérer des stratégies telles la migration ou l'émulation afin d'assureur la pérennité de l'œuvre. La migration consiste à faire passer une technologie de support de contenu vers une autre, plus récente, afin d'assurer la possibilité de faire fonctionner ce contenu avec les équipements de la technologie plus récente. L'exemple le plus banal aujourd'hui est le passage de la bande vidéo analogique à un support numérique. L'émulation quant à elle est beaucoup plus complexe. Cette approche cherche à préserver l'efficacité opérationnelle de l'œuvre en recréant exactement les mêmes effets technologiques à l'aide de moyens plus récents. Elle est donc beaucoup plus spécifique.

Mais quels sont les critères qui nous permettent de prendre une telle décision, en dehors du fait que les technologies sont vouées à être remplacées? Quelles sont les œuvres qui devront être émulées plutôt que migrées? N'y-a-il pas des œuvres qui par leur nature conceptuelle ne peuvent exister que selon leur format technologique original sous peine d'entamer leur signification? Pip Laurenson, restauratrice principale des nouveaux médias à la Tate Modern de Londres, propose d'aborder la question de la conservation des équipements technologiques en mesurant leur importance dans l'œuvre par rapport à trois valeurs : esthétique, conceptuelle et historique. Laurenson lie la valeur esthétique strictement à la fonctionnabilité de l'équipement, c'est-à-dire aux capacités techniques de transmettre adéquatement le son, l'image ou l'interaction souhaitée inscrite sur un support de contenu. Il s'agit donc d'apprécier la qualité du rendu de l'expérience de l'œuvre par ses équipements. La valeur conceptuelle établit le lien que la technologie entretient avec la signification de l'œuvre et les intentions de 
l'artiste. Si le lien conceptuel de la technologie utilisée est important pour le sens de l'œuvre, il sera difficile d'admettre la migration de cette technologie, malgré le danger d'obsolescence. La valeur historique situe ces équipements sur une trajectoire temporelle liée à l'histoire des technologies des médias, mais aussi à l'inscription historique de l'cuvre dans le corpus de production de l'artiste. La mesure comparative de ces trois valeurs permettra de déterminer le protocole de préservation qu'il faudra favoriser. L'une des grandes qualités de ce modèle décisionnel est de ne pas perdre de vue l'ancrage historique des cuvres à contenu technologique. Et, à mon avis, cela pose un enjeu de taille pour les intervenants qui collectionnent ces œuvres.

Nous assistons fort probablement en ce moment à un tournant technologique. Le mode analogique est toujours présent, mais la disponibilité des équipements devient de plus en plus rare, donnant prépondérance au mode numérique. Dans 20 ans, le mode analogique risque d'avoir complètement disparu commercialement, laissant les œuvres analogiques à la responsabilité des institutions et des artistes, les localisant dans un contexte historique délimité. Cet historique des technologies est documenté de façon fragmentaire en raison de la rapidité des changements et le peu de recul dans lequel elles s'inscrivent. Idéalement, certaines œuvres jugées importantes et sans doute appartenant à des collections devraient être non seulement bien documentées, mais maintenues dans leur technologie initiale dans l'espoir de conserver des œuvres qui témoignent de l'usage de ces technologies et d'en permettre l'expérience.

Cet aspect de l'expérience amène à mettre une autre considération en relation avec nos trois valeurs d'analyse et pouvant conséquemment influencer la stratégie de préservation. Il s'agit de la présence physique de l'équipement dans la mise en espace, le fait qu'il soit exposé ou non à la vue du spectateur. En effet, l'équipement peut être utilisé uniquement à des fins de fonctionnement et pour ses capacités techniques. Il est alors dissimulé à la vue du spectateur, intégré à des éléments sculpturaux, placé derrière la cloison des murs ou du plafond. Il n'a aucune influence sur les caractéristiques 
visuelles et sémantiques de l'œuvre, contrairement à cet autre régime d'usage où il est clairement en vue. On peut apprécier que, selon cette dernière situation, l'équipement risque d'avoir une valeur marquée non seulement sur l'aspect conceptuel, mais aussi historique. De même, la dimension esthétique sera sous influence car après tout c'est potentiellement un objet sculptural avec son volume, une forme physique et un traitement de matière.

La collection du Musée des beaux-arts de Montréal possède une œuvre de Jacques Perron intitulée Défilement (1997) (ill. 6) qui relève bien de cette problématique. Il s'agit d'une monobande VHS qui a été transférée en Bétacam. L'équipement, un lecteur $C D$ correspondant au format de la copie d'exposition, est toujours dissimulé derrière la cloison. La présentation de l'image se fait sur un petit moniteur vidéo posé sur un socle de 122 centimètres de haut dont la surface correspond à l'empreinte du moniteur, donnant une allure monolithique à l'ensemble. Le socle est à bonne distance du mur de la salle, de façon à affirmer le caractère sculptural de l'œuvre. Le moniteur vidéo dont le boîtier est en acier, de facture industrielle, est un modèle utilisé habituellement en salle de montage. La bande présente une séquence d'images où un œil, en très gros plan, défile de gauche à droite et en retour de droite à gauche, avec passage délibéré de cadrages. On imagine que c'est le même œil fixe, mais l'image est en fait le résultat complexe et subtil de multiples superpositions de saisies d'un cil laissant la définition finale de l'image incertaine, suspendue. Perron pose la question des limites de la photographie et en quoi celle-ci peut encore exister dans l'image vidéographique. Une attention est portée au grain de l'image versus sa composition vidéo pulsante, d'autant plus que l'image est perçue à travers une feuille de papier blanc maintenue en place devant l'écran uniquement par le statisme produit par le moniteur à tube cathodique (CRT). Cette feuille ne peut tenir en place qu'à cause de la charge électromagnétique produite par le tube cathodique. Il semble douteux que des écrans plus récents tels ceux de type LCD (écran à cristaux liquides) puissent produire la même charge électromagnétique. On le voit, le projet tourne autour de l'image 
analogique et un certain effet de l'œuvre - le maintien de la feuille - est conceptuellement fortement lié à ce type d'écran. Non seulement la valeur conceptuelle de l'équipement est primordiale, mais on observe que la valeur esthétique du moniteur l'est tout autant. C'est au cours de l'entretien mené auprès de l'artiste que cette dernière importance est clairement apparue. En première partie, Perron affirmait qu'il ne voyait pas de problème avec la possibilité de changer le moniteur pour un modèle semblable, en autant que ce soit un écran qui maintienne le statisme. Du même coup, il ne cessait d'insister sur le fait que l'aspect monolithique anthropomorphique de la combinaison socle-moniteur était très important. 11 a même qualifié la présentation de sculpturale et a dit l'avoir pensée comme telle. Au fil de l'entretien, non seulement le lien de la technologie avec le projet conceptuel a-t-il été démontré, mais la qualité sculpturale, c'est-à-dire les dimensions, l'apparence physique du moniteur, est apparue tout aussi importante. La conséquence pour le musée est d'assurer la pérennité de ce moniteur par un programme régulier d'entretien et l'achat de un ou deux moniteurs supplémentaires du même modèle.

Un autre cas qui dénote la présence esthétique avec la mise en vue de l'équipement est l'œuvre sonore Bound Voices (19971998) (ill. 7) de Betty Coodwin, qui a cependant des conséquences quelque peu différentes sur le plan de la pérennité. Elle est composée d'une longue trainée de fils métalliques conducteurs entremêlés, maintenue à la verticale à distance du mur par une structure triangulaire en bois peinte en noir. A différentes hauteurs sur cet entremêlement, on distingue une série de petits haut-parleurs radio désossés, desquels on entend faiblement différentes voix humaines. L'équipement qui permet l'émission sonore est composé d'un lecteur $C D$ noir, représentant ces premières générations d'appareil compact Sony, déposê sur un module d'amplification au sol, à la gauche des fils suspendus, tout près du mur. Ce lecteur a pris de l'importance pour l'artiste en raison de sa couleur, de sa forme et de sa miniaturisation. L'œuvre fut acquise par le Musée d'art contemporain de Montréal qui, au moment de l'acquisition, n'a pas jugé nécessaire de se procurer un lecteur compact similaire, ces 


$$
1
$$




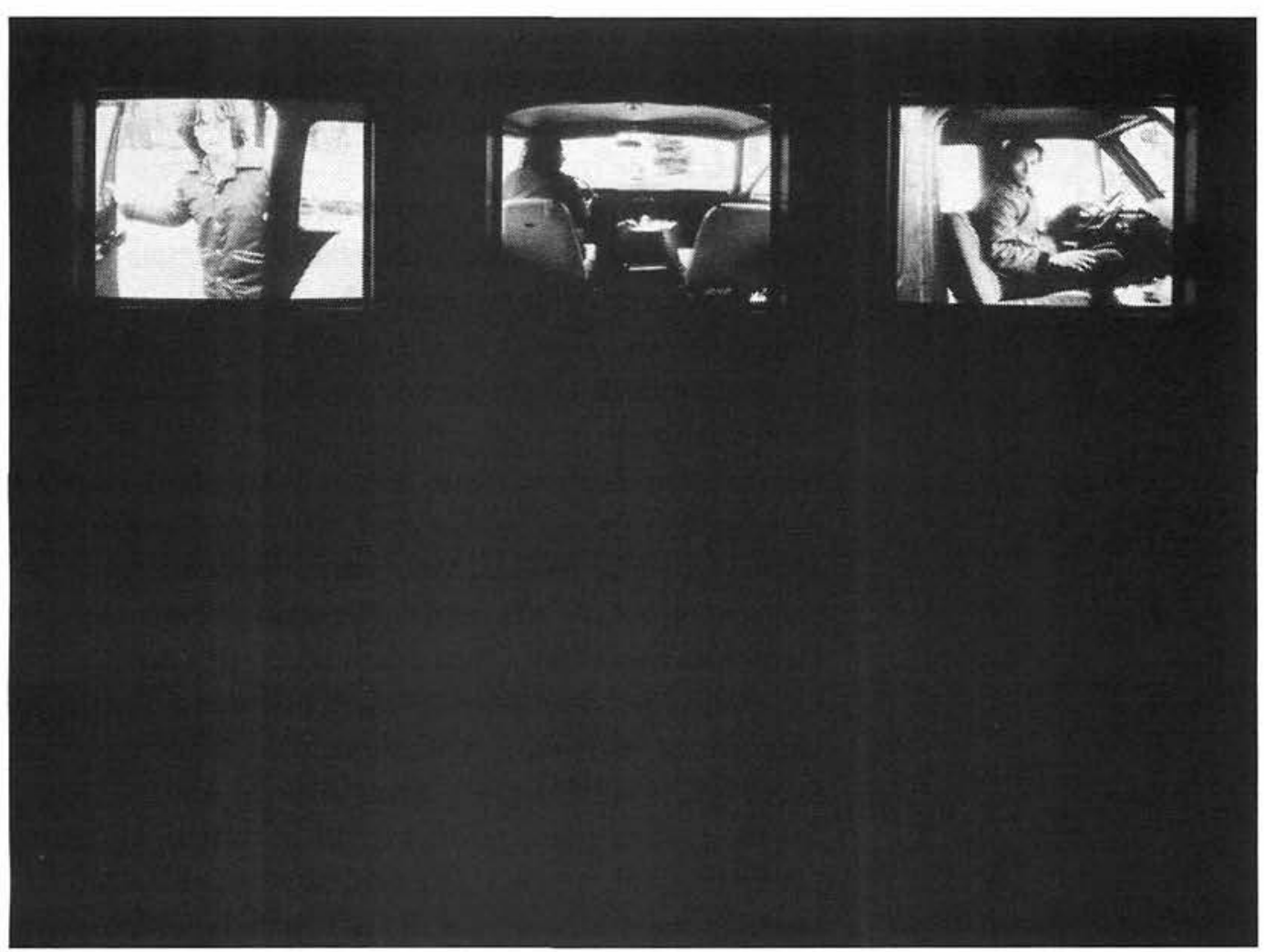

\section{Illustration 7}

Betty Goodwin, Bound Voices, 1997-1998, acier, fil métallique, plomb, bois, boîtier d'amplification, lecteur $C D$, disque compact, haut-parleurs et filage électrique, $235 \times 85 \times 34.3 \mathrm{~cm}$. @ Musée d'art contemporain de Montréal. Photo: Richard-Max Tremblay, avec l'aimable permission de la Calerie René Blouin

\section{Illustration 8}

John Massey, As the Hammer Strikes (A Partial Illustration), 1982, un film $16 \mathrm{~mm}$ en couleurs et deux films $16 \mathrm{~mm} \mathrm{n} / \mathrm{b}, 30 \mathrm{~min}$ chacun, avec trois projecteurs et système de synchronisation. Musée des beaux-arts du Canada, Ottawa. Photo: $\odot \mathrm{MBAC}$ 
instruments affichant une bonne durabilité. Cependant, l'appareil a rapidement présenté des problèmes de fonctionnement et il s'avéra qu'il était impossible de le réparer. On a essayé de trouver sur le marché un autre lecteur de même modèle, mais sans succès. En consultation avec l'artiste dans le but de localiser un modèle de remplacement, celle-ci a insisté sur le fait que ce lecteur, par ses caractéristiques, faisait partie de l'esthétique de l'installation, d'où l'impossibilité de lui trouver un remplaçant d'une autre facture. La solution proposée fut de trafiquer l'intérieur du boîtier du CD et d'introduire un lecteur de type MP3 à la place de son mécanisme de lecture laser, tirant ainsi partie d'une technologie plus récente, heureusement encore plus compacte, et capable de diffuser de même manière le contenu de l'œuvre sans modifier l'apparence visuelle de l'installation. Le contenu de l'œuvre devient un fichier numérique et n'a plus comme tel de support physique. C'est donc à une émulation que la technologie de Bound Voices fut soumise. Ironiquement, l'expression technologique historique de l'œuvre est maintenue, même si elle perd sa fonction opératoire, contrairement à l'exemple de l'œuvre de Jacques Perron. C'est que sa valeur esthétique prône, d'où la persistance du boîtier compact de type $C D$.

On le voit, ces deux cas de figure montrent à l'analyse des valeurs proposées par Pip Laurenson que leur maintien peut parfois admettre une certaine perte sans pour autant entamer de façon cruciale l'intégrité générale de l'œuvre.

\section{Justine Lebeau}

Les cas de Bound Voices et de Tap montrent bien en quoi la migration ou l'émulation viennent modifier la nature technologique originale des équipements et des supports de contenu. Pourriez-vous nous parler davantage de cette capacité à assurer la pérennité des cuuvres à contenu technologique en privilégiant ces stratégies ?

\section{Richard Cagnier}

Ce que la migration et l'émulation provoquent, créant un certain malaise pour les muséologues dans leur processus de conservation de l'œuvre à contenu technologique, c'est 
la perte du matériau original. Ce transfert est une perte si on analyse l'œuvre sous l'angle du concept d'authenticité tel que Brandi le propose. Cette perte n'est pas définitive si l'on parvient à maintenir les composants originaux de l'œuvre en bonne condition, mais, au fil du temps, la possibilité de rejouer l'ceuvre selon sa technologie d'origine s'amenuise. D'où l'importance de bien décrire et de documenter, par captation vidéographique et photographique si nécessaire, les effets de l'cuvre et ses comportements, de façon à pouvoir assurer le maintien de son intégrité. Mais il y a des cas où cette technologie d'origine a toujours causé un problème et où le relais de la migration ou de l'émulation sont littéralement des approches salvatrices.

L'œuvre de John Massey As the Hammer Strikes (1982) (ill. 8) de la collection du Musée des beaux-arts du Canada pose très justement cet enjeu de la migration. Ce cas démontre qu'elle permet d'assurer non seulement la pérennité de l'œuvre, mais sa possibilité d'itération récurrente qui, dans ce cas, n'était pas assurée. En fait, l'œuvre de Massey a fini par établir son mode de présentation "correcte" 25 ans après qu'elle ait été créée, justement par la migration des supports de contenu. Au départ, l'œuvre est en format filmique $16 \mathrm{~mm}$. Elle consiste en une projection synchrone de trois images de mêmes dimensions présentées côte à côte sur un mur, à distance très rapprochée. L'image du centre, en couleur, présente deux individus, l'un conduisant une camionnette qui fait monter un autostoppeur au début du film. S'ensuivra une conversation informelle entre les deux protagonistes. Ce long plan fixe est pris de l'intérieur de la camionnette, la caméra étant placée derrière les deux hommes. Les deux autres projections, en noir et blanc, présentent toutes sortes d'images liées somme toute à la conversation, mais les séquences semblent plongées dans la psyché respective des deux individus : côté gauche, le conducteur, côté droit, l'auto-stoppeur qui présente des difficultés de langage. Cette cuvre a été créée dans le cadre de l'exposition O'Kanada présentée à Berlin en 1982, dont le cornmissariat avait été confié à Pierre Théberge. John Massey est un artiste conceptuel; il sélectionne les médiums qui lui semblent appropriés dépendant des idées et des concepts 
qu'il veut explorer. Il a utilisé autant la photographie, le film que le son ou la lumière, souvent en conjonction avec des objets. Dans l'ensemble de sa production, la photographie est prédominante et c'est le travail sur l'image qui caractérise sa production. L'enjeu technique de As the Hammer Strikes est sans doute de réussir la parfaite synchronisation des trois films d'égale durée, de trente minutes. La technologie des boucleurs n'étant pas très répandue à l'époque de la création de l'œuvre, Massey a alors pensé à poser sur une table trois projecteurs qui étaient entraînés l'un à l'autre à l'aide d'un système de courroies de caoutchouc liées aux trois moteurs, permettant en principe de maintenir la vitesse des projecteurs constante et donc la simultanéité des trois projections. Ces projecteurs étaient installés dans une salle de projection fermée, située à l'arrière de la salle de présentation. $\AA$ Berlin, très rapidement le système a montré des défaillances, si bien que les projections étaient interrompues. A ma connaissance, lorsque l'exposition s'est terminée, la pièce de Massey n'était plus projetée depuis un certain temps. Une version de la même exposition a été présentée au Musée des beaux-arts de Montréal un an après l'exposition de Berlin, On a tenté de représenter As the Hammer Strikes sous forme filmique et le même problème d'incapacité à assurer le synchronisme des projections s'est produit. Malgré ses problèmes technologiques non résolus, l'œuvre a toutefois été acquise quelques années plus tard par la conservatrice de l'art contemporain au Musée des beaux-arts du Canada, en raison de son importance dans le développement du mouvement conceptuel canadien et dans l'œuvre de Massey. Le musée a assumé le fait que le problème de synchronisme devait être résolu.

L'œuvre a été dormante jusqu'à ce que Jean Gagnon, conservateur des arts médiatiques au MBAC, ait été approché en 1992 par Ihor Holubizky, conservateur à la Galerie d'art de Hamilton, au sujet de As the Hammer Strikes. Il organisait une exposition solo de l'artiste et voulait inclure cette œuvre. Gagnon a pensé à sa migration sous forme vidéographique. Le grand avantage avec cette migration, c'est que la simultanéité des projections serait facile à établir, l'existence de contrôleur électronique pour projection 
multiple vidéographique étant disponible. Encore fallait-il justifier cette migration. En discussion avec l'artiste, celuici a affirmé que le support filmique n'était pas essentiel à l'œuvre parce que c'était la valeur conceptuelle - la structure du scénario, le dialogue et les images en simultanée qui était prépondérante, et le fait que les trois projections, quelle que soit leur source, soient de mêmes dimensions. L'œuvre a d'abord été transférée sur bande Bétacam SP afin d'assurer la copie sous-maitresse de l'œuvre. Pour la copie d'exposition, on a utilisé le disque laser, alors très courant au milieu des années 1990. C'est ainsi que l'œuvre fut présentée avec succès à Hamilton, mais aussi subséquemment à Toronto à la fondation Ydessa Hendeles. Plus tard, en 2006, l'œuvre fut demandée pour son inclusion dans le projet d'exposition Beyond Cinema: the Art of Projections qui s'est tenue au Hamburger Bahnhof à Berlin. L'artiste a souhaité aller plus loin que le contenu analogique parce que la technologie des disques laser est devenue elle aussi obsolète à la fin des années 1990. Il a fallu numériser les bandes sousmaîtresses Bétacam SP. L'œuvre a donc subi un second transfert technologique, passant de l'analogique au numérique, et est aujourd'hui sur DVD. Il semble qu'à partir de cette dernière présentation la version "correcte" de l'œuvre telle que Genette l'a définie ait enfin été établie, tant sur le plan technologique qu'en ce qui concerne les prescriptions de présentation de l'œuvre, par exemple en établissant la dimension des images.

On peut voir que le musée a assuré la pérennité de l'objet non seulement en termes de responsabilité de préservation-conservation, mais aussi dans la capacité de promouvoir son usage. On a souvent tendance à associer le musée avec le mausolée où l'œuvre y est déposée, protégée, mais de moins en moins "activée" pour reprendre les mots de Nelson Goodman'. Pour As the Hammer Strikes, le musée a pleinement assumé sa responsabilité de médiateur en assurant d'abord la protection des sources de l'œuvre pour ensuite élaborer les conditions pouvant assurer sa manifestation et ce, de façon récurrente.
5

GOODMAN, Nelson. Langages de l'art: une approche de la théorie des symboles. Nimes: Editions Jacqueline Chambon, coll. * Rayon art $1992,312 \mathrm{p}$. 